\title{
ORGANIZAÇAO DO XXVI CONGRESSO BRASILEIRO DE ENFERMAGEM
}

PRESIDENTES DE HONRA

Sua Exa. o Senhor ERNESTO GEISEL

Presidente da República Federativa do Brasil

Sua Exa. o Senhor NEY AMINTHAS DE BARROS BRAGA Ministro da Educação e Cultura

Sua Exa. o Senhor PAULO DE ALMEIDA MACHADO Ministro da Saúde

Sua Exa. o Senhor ARNALDO CESAR PRIETO Ministro do Trabalho

Sua Exa. o Senhor LUIZ GONZAGA DO NASCIMENTO E SILVA Ministro da Previdência Social

HOMENAGEM ESPECIAL

Sua Exa. o Senhor EMILIO HOFFMANN GOMES

Governador do Estado do Paraná

Sua Exa. o Senhor IVAN BEIRA FONTOURA

Secretário de Estado dos Negócios de Saúde Pública do Paraná

Sua Exa. o Senhor JAIME LERNER

Prefeito Municipal

HOMENAGEM

Sua Exa. o Senhor JAYME CANET JUNIOR

Vice-Governador do Estado do Paraná

Sua Exa. o Senhor PAULO FRANILO TORRES

Presidente do Senado Federal

Sua Exa. o Senhor CELIO BORBA

Presidente da Câmara dos Deputados

Sua Exa. o Senhor ROBERTO SANTOS

Presidente do Conselho Federal de Educação

Sua Exa. o Senhor ERNESTO KNAUER

Presidente do Conselho Estadual de Educação do Estado do Paraná

Sua Exa. o Senhor CANDIDO MANOEL MARTINS DE OLIVEIRA

Secretário de Educação e Cultura do Estado do Paraná 
Sua Exa. o Senhor THEODÓCIO JORGE ATHERINDO Magnífico Reitor da Universidade Federal do Paraná

Sua Exa. o Senhor OSWALDO ARNS Magnífico Reitor da Universidade Católica do Paraná

Sua Exa. o Senhor OSCAR ALVES

Magnífico Reitor da Fundação Universidade Estadual de Londrina

Sua Exa. o Senhor SAMUEL AUGUSTO C. CORREA Comandante da 5. ${ }^{a}$ Região e 5. ${ }^{a}$ Divisão de Infantaria

Sua Exa. o Senhor NELSON DIAS DE SOUZA MENDES Comandante da Escola de Oficiais Especialistas e de Infantaria de Guarda

Sua Exa. o Senhor CEZAR PASSO SALDANHA LEMOS Comandante da Polícia Militar do Estado

Sua Exa. o Senhor DOM PEDRO FEDALTO Arcebispo Metropolitano

Sua Exa. o Senhor BOLESLAU USSYK Delegado Federal de Saúde da 10. ${ }^{\text {a }}$ Região

Sua Exa. a Senhora BEILA RAIZ Diretora do Departamento de Saúde da Secretaria de Saúde Pública

Sua Exa. o Senhor JOAO MANSUR Presidente da Assembléia Legislativa do Estado do Paraná

Sua Exa. o Senhor EDGARD DANTAS PIMENTEL Presidente da Câmara Municipal de Curitiba

COLABORADORES DO XXVI CONGRESSO

BRASILEIRO DE ENFERMAGEM

Dr. Ewaldo Nickel

Associação dos Profissionais Liberais Universitários do Brasil APLUB

Universidade Católica do Paraná

Universidade Federal do Paraná

Faculdade de Educação Universitária Federal do Paraná

Faculdade de Ciências da U.F.PR.

Escola de Enfermagem "Madre Leonie"

Escola Técnica de Enfermagem "Catarina de Labouré"

Escola Evangélica de Auxiliares de Enfermagem

Escola de Auxiliares de Enferma€gm "Dr. Caetano Munhoz da Rocha" 
Hospital de Clínicas da U.F.PR.

Hospital Pinheiros

Eletro Médica

Casa Lapalu

Seção de Enfermagem do Hospital de Clínicas da U.F.PR.

Móveis Cimo S/A

Prosdócimo S/A

Café Cacique

Gazeta do Povo

O Estado do Paraná

Diário do Paraná

TV Iguaçú Canal 4

TV Paraná Canal 6

TV Paranaense Canal 12

Rádio Iguaçú

Companhia de Telecomunicações do Paraná - TELFPar

Empresa Paranaense de Turismo - PARANATUR

Viação Aérea Riograndense - VARIC

Indústria de Papel Simão $\mathbf{S} / \mathbf{A}$

Banco do Estado do Paraná

Governo do Estado

Prefeitura Municipal

Bonato \& Companhia Limitada - Lembranças do Paraná

Polícia Militar do Estado

Ginásio de Desportos do Tarumã

Johnson \& Johnson - Divisão Hospitalar

Ethicon Suturas S/A

Darrow Laboratórios $\mathbf{S} / \mathbf{A}$

Cyanamid Química do Brasil Ltda - Divisão Davis-Gek

The Sydney Ross Co.

Creemer S/A - Produtos Texteis e Cirúrgicos

York S/A Indústria e Comércio

Mimex - Mercantil Importadora Exportadora Ltda

Pfizer - Divisão Farmaceutica

Casa Provincial das Filhas de Caridade

Santa Mónica Club de Campo

Secretaria de Saúde

Serviço Nacional de Aprendizado Comercial - SENAC

Hermes Macedo S/A

FUNDEPAR

Imprensa Oficial do Estado do Paraná

Secretaria da Agricultura 
Terumo Brasil S/A - Comercial Importação

Laboratório Miles do Brasil

Maison Demy

Correios e Telégrafos do Estado do Paraná

DIREÇAO DO XXVI CONGRESSO BRASILEIRO DE ENFERMAGEM COMISSOES:

COMISSAO DE COORDENAÇAOO

Dra. Glete de Alcântara - Presidente

Maria da Graça Simões Côrte Imperial - 1.a Vice-Presidente

Dra. Amália Corrêa de Carvalho - 2.a Vice-Presidente

Taka Oguisso - Tesoureira

Clarice Ferrarini - Secretária Executiva

COMISSAO DE TEMAS

Dra. Circe de Melo Ribeiro - Coordenadora

Dinéa Tognini

Kimie Ando

Irmã Francisca Teixeira

Neyde Goés Benuthe

COMISSAO DE RECOMENDAÇOES

Maria José Schimidt

Vitória Secaf

Vilma Balielo

Antonia Xaiver da Silva

COMISSAO EXECUTIVA

Alice de Lima - Coordenadora

Glaucia Borges Seraphim - Secretária

Neusa Aparecida Ramos - Tesoureira

Maria Leda Vieira - Assessora

Terezinha Beatriz G. de Azeredo - Assessora

SUB-COMISSOEES

FINANÇAS

Neusa Aparecida Ramos - Coordenadora

Irmã Maria Magrin

Irmã Fagundes

Rita Faraco

\section{DIVULGAÇÃO}

Irmã Alides Breda - Coordenadora

Luzia Nahoyo Oka 
Filomena Tartas

Elizabeth Sens

Irmã Marinês Bavaresco

\section{RECEPÇAO}

Irmã Rosalinda Risson - Coordenadora

Valmir Terezinha Kehrwald

Luci Angélica S. Marques

Aloma Justus

Renate Henrinchs

HOSPEDAGEM

Gerda Mitt - Coordenadora

Irmã Maria Turkiewcz

Maria Rocha Lima

PREPARO DO LOCAL

Ruth Tulio - Coordenadora

Dirce Oliveira Nascimento

Glória Nicoladeli

Yaci de Castro Vaz

Gudrun Ingrid Dueck

Jussara Cavaglieri

Lilian Deisy Woiski

SOCIAL

Eliana Portella Carzino - Coordenadora

Leila Araujo Cruz

Ivonete S. G. Kulak

Maria da Graça Ventura

Benedita R. Azeredo

REGISTRO

Thereza Meiga Pinto - Coordenadora

Maria do Socorro Gerum

Kioko Yakama

Milton N.orais

Filomena P. de Almeida

Walmy Schmidt

Rita Otsuka

Clarisse Neves

Lúcia Toyoshima Krauser

Bernardete Charvet

Tereza Suzi Betega Pinto 
MONITORIA

Josefá Lourenço Meira - Coordenadora

Irmã Otilia Junges

Clarita Chiodi

Helena S. Mota

SECRETARIA

Glaucia Borges Seraphim - Coordenadora

Carmela Santos Martins

Yoshie Almeida

PROGRAMA CIENTIFICO

Endereço:

Faculdade de Educação

Rua General Carneiro, 460

\section{TEMAS OFICIAIS}

TEMA I - Necesidades Psicossociais e psicoespirituais dos pacientes Simpósio

Segunda-feira - dia 15 de 14 às 18 horas

Auditório

Presidente da Sessão: Neusa Aparecida Ramos

Membros: Dra. Amélia Tereza M. Vasconcelos (HSESP)

Pe. Ivo Gelain

Lygia Paim (EEUF'RJ)

TEMA II - Liderança

Conferência

Terça-feira - dia 16 de 8 às 12 horas

Auditório

Conferencista: Prof. Dr. Bachir Haidar Jorge

Discussão em Grupo

Terça-feira dia 16 de 14 às 18 horas

Áreas

Anf. 1 - Direção do Serviço de Enfermagem

Coordenadora: Maria Camargo de Oliveira Falcão

Anf. 2 - Chefia de Unidade de Enfermagem

Coordenadora: Clarice Oliveira

Irmã Francisca Nogueira

Anf. 3 - Cursos de Pós-Graduação em Enfermagem

Coordenadora: Dra. Amália Corrêa de Carvalho

Assessora : Vilma de Carvalho 
Anf. 4 - Cursos de Graduação e habilitação em Enfermagem Coordenadora: Dra. Maria Aparecida Minzoni Assessora : Nilza da Rocha Medeiros

Anf. 5 - Cuidados en? Terapia Intensiva Coordenadora: Dra. Wanda de Aguiar Horta Assessora : Dra. Haydée Guanais Dourado

TEMA III - Recentes pesquisas em Enfermagem Anfiteatro 1 Quarta-feira - 17-7 de 8 às 12 horas Presidente da Sessão María Helena Nery

Trabalhos:

1 - Avaliação da dor em pacientes submetidos à cirurgia de tireóide pela aplicação do método de escala diferencial semântica de Osgood.

Autora: Dra. Wanda de Aguiar Horta (EEUSP)

2 -- Aerosolterapia em asmáticos - uma observação de enfermagem.

Autora: Maria Pitol Rodrigues (HC.FMUSP)

3 - A propósito da Classificação Brasileira Uniforme de Ocupaçōes Autora: Taka Oguisso (INPS-SP)

4 - Influência da ingestão alimentar no hemograma Autora: Marcia Karon Rufino (EE Ribeirão Preto)

5 - Experiência em Auditoria de Enfermagem Autora: Paulina Kurcgant (EEUSP)

6 - Padrões mínimos nos hospitais contratados pelo INPS na $\mathrm{Ca}$ pital de São Paulo

Autora: Odette Aparecida de Carvalho Bocchi (INPS-SP)

7 - Obtenção de urina em jato com assepsia - Estudo experimental Autora: Dra. Emilia L.S. Angerami (EE Ribeirāo Preto)

TEMA III - Recentes pesquisas em Enfermagem

Anfiteatro 2

Quarta-feira - 17-7 de 8 às 12 horas

Presidente: Vitória Secal

Trabalhos:

1 - Estudo do aparecimento do hábito de pica e desejos em gestantes Autora: Maria Solange G. Tavares (EE Ribeirão Preto)

2 - Estudo comparativo da secreção orofaringea em duas populações distintas

Autora: Branca Maria de Oliveira (EE Ribeirão Preto) 
3 - Avaliação da aplicação do histórico de enfermagem por estudantes da EEUSP

Autora: Dra. Wanda de Aguiar Horta (EEUSP)

4 - Estudo bacteriológico das úvulas do estetoscópio Autora: Dra. Emilia L. S. Angerami (EE Ribeirão Preto)

5 - Evolução da temperatura axilar e retal de recém-nascidos normais nas primeiras 48hs. de vida.

Autora: Maria Lucia C. Santos (EE Ribeirão Preto)

$\boldsymbol{\epsilon}$ - Análise da rotina de verificação de sinais vitais do paciente no pós-operatório, em um centro de recuperação.

Autora: Ana Maria Palermo da Cunha (EE Ribeirão Preto)

7 - Atividade de enfermagem nos ambulatórios de um hospital governamental.

Autora: Dra. Maria Inês Ribeiro de O. Cicinelli (EE Ribeirão Preto)

8 - Estudo preliminar sobre o efeito das aplicações frias em pacientes com febre de origem infecciosa.

Autora: Dra. Yoriko Kamiyama (EEUSP)

TEMA IV - Educação em Enfermagem

Anfiteatro 3

Quarta-feira - 17-7 de 8 às 12 horas

Presidente da Sessão: Dra. Haydée Guanais Dourado

Trabalhos:

1 - Formação do pessoal de enfermaegm no Brasil Autora: Nylza da Rocha Dias de Medeiros (GB)

2 - Relato da experiência do $1^{\circ}$ ano do Curso de Residência para Enfermeiras na EE e Hospital prof. Edgar Santos da UF da Bahia

Autora: Maria Helia de Almeida (UFB - Salvador)

3 - Atualização do ensino de tica.

Autora: Dra. Francisca Nogueira Soares (EPE S. Paulo)

4 - Uma experiência sobre Programação do Ensino por Objetivo Autora: Ir. Maria Turkievwicz (ETE Catarina de Labouré Paraná)

TEMA V - Aspectos assistenciais de Enfermagem Anfiteatro 4

Quarta-feira - 17-7 de 8 às 12 horas

Presidente da Bessão: Herdy de Almeida Vieira 
Trabalhos:

1 - Assistência de Enfermagem à família no Hospital do Servidor Público Estadual.

Autora: Dra. Juana Lopez de La Pena (HSPE - SP)

2 - Planejamento de cuidados na Unidade de Terapia Intensiva Autora: Alice Martins Gomes (H. Sírio Libanes - SP)

3 - Assistência de Enfermagem aos pacientes na artroplostia total do quadril

Autora: Terezinha Damante da Silva (HSPE - SP)

4 - Assistência de Enfermagem a pacientes portadores de Moléstia de Wilson

Autora: Ester Ferreira Silva (HC. FMUSP)

5 - Pensando em Psiquiatria Comunitária Autora: Antonia Regina F. Rodrigues (EE Ribeirão Preto)

6 - Ventilação Pulmonar - alguns aspectos de enfermagem relacionados a pacientes com aparelho de respiração artificial Autora: Clarice M. Higuchi (HC. FMUSP - DOT)

7 - Nossa experiência com pacientes em insuficiência respiratória Autora: Edna Rodrigues (HC FMUSP)

8 - "Amparo Maternal" - Campo de Estágio de Enfermagem Obstétrica

Autora: Adareluce Matta Periotto (H.S. S. Paulo)

TEMA VI - Problemas de Infecção Hospitalar Anfiteatro 5

Quarta-feira - 17-7 de 8 às 21 horas

Presidente da Sessão: Vilma Balielo

Trabalhos:

1 -- Atuação do pessoal de Enfermagem nas medidas técnico administrativas de controle das infecções hospitalares.

Autora: Celina da Cunha Tibiriçá (Lab. Winthrop RGS)

2 - Uma experiência de enfermagem no controle da infecção hospitalar.

Autora: Neyde Góes Benuthe (DOT-HC FMUSP)

3 - Ar condicionado em ambientes assepticos. Autora: Marilda Aparecida Pierini (HC FMUSP)

1 - Estudo da utilização das soluções desinfetantes em C.C. em alguns hospitais do Município de $\mathbf{S}$. Paulo

Autora: Vanda M.G. Jouclas (EEUSP)

Quinta-feira - 18-9 de 8 às 16 horas

Visita à Instituições Sociais e de Saúde 
TEMAS LIVRES

Área 1 - Hospital

Anfiteatro 1

Sexta-feira - 19-7 de 8 às 12 horas

Presidente: Maria Camargo de Oliveira Falcão

Trabalhos:

1 - Atividades de Enfermagem em Otoneurologia.

Autora: Lucira F. Vasconcelos (HC FMUSP)

2 - Assistência de Enfermagem ao paciente que vai se submeter a estudo hemodinâmico

Autora: Maria Samitaro Kalen (Inst. de Cardiologia - SP)

3 - O trabalho da Enfermeira na Unidade de Hemodiálise. Autora: Léia Alvares

4 - Aspectos físicos e psicológicos do paciente queimado.

Autora: Carmem Lúcia Sfair (HC FMUSP)

Área II - Comunidade

Anfiteatro 2

Sexta-feira - 19-7 de 8 às 12 horas

Presidente: Pe. Ivo Gelaín

Trabalhos:

1 - Atuação da Enfermeira na liga de combate à Sifilis Autora: Edina Maria Teixeira de Carvalho (HC FMUSP)

2. - Subsídios para instalação de um serviço para acidentados do trabalho

Autora: Maria José Schmidt (INPS-SP)

3 - Organização de um Serviço de Enfermagem de Saúde Pública no Estado de Sta. Catarina

Autora: Ingrid Elsen (Sta Catarina)

4 - A Enfermagem no Estado do Paraná - Subsídios

Autora: Dra. Wanda de Aguiar Horta (EEUSP)

5 - Das possibilidades de um melhor entrosamento entre Hospital - Educação e Comunidade

Autora: Maria Bernadeth Santos (CS Raphael de Paula Souza

- HE - RJ)

Área III - Organizaçāo

Anfiteatro 3

Sexta-feira - 19-7 de 8 às 12 horas

Presidente da Sessão: Paulina Kurcgant 
Trabalhos:

1 - Organização de um ambulatório Psiquiátrico Autora: Toyoko Saiki

2 - Análise de uma éxperiência em tretnamento de pessoal auxiliar de enfermagem.

Autora: Dra. Maria Aparecida Minzoni (EE Ribeirão Preto)

3 - Controle de variáveis num grupo de atendentes em treinamento Autora: Dra. Maria Aparecida Minzoni (EE Ribeirão Preto)

4 - Estudo prévio sobre integração de Enfermagem em Centro Cirúrgico e Enfermagem Cirúrgica.

Autora: Dra. Sonia D. T. Salzano (EEUSP)

Área IV - Outros

Anfiteatro 4

Sexta-feira - 19-7 de 8 às 12 horas

Presidente da Sessão: Lourdes Góes Müller

Trabalhos:

1 - Atualização de técnicas de Enfermagem em cirurgia transuretrais

Autora: Marilda A. Pierini (HC FMUSP)

2 - Experiência de enfermagem com o dialisador Kill no Instituto de Cardiologia do Estado

Autora: Maria José Dantas (Inst. Cardiologia SP)

3 - Avaliação das atividades de Enfermagem desenvolvidas no periodo da tarde

Autora: Laurinda M. Gandolla (HC FMUSP)

4 - Bases para uma ciência de Enfermagem Autora Dra. Wanda A. Horta (EEUSP)

5 - Um estudo sobre a personalidade do estudante de Enfermagem de nível médio

Autora: Nelly de Matos Mehl (ETE - Paraná)

6 - Histórico e aspectos legais da adoção no Brasil

Autora: Dilce Rizzo Jorge (EEUSP)

Sábado - 21-7 de 8 às 11 horas

Sessão Plenária 